\title{
Low friction thermoplastic polyurethane coatings imparted by surface segregation of amphiphilic block copolymers
}

Røn, Troels; Javakhishvili, Irakli; Jeong, Seonghyeon; Jankova, Katja; Lee, Seunghwan

Published in:

Colloid and Interface Science Communications

Link to article, DOI:

10.1016/j.colcom.2021.100477

Publication date:

2021

Document Version

Publisher's PDF, also known as Version of record

Link back to DTU Orbit

Citation (APA):

Røn, T., Javakhishvili, I., Jeong, S., Jankova, K., \& Lee, S. (2021). Low friction thermoplastic polyurethane coatings imparted by surface segregation of amphiphilic block copolymers. Colloid and Interface Science Communications, 44, [100477]. https://doi.org/10.1016/j.colcom.2021.100477

\section{General rights}

Copyright and moral rights for the publications made accessible in the public portal are retained by the authors and/or other copyright owners and it is a condition of accessing publications that users recognise and abide by the legal requirements associated with these rights.

- Users may download and print one copy of any publication from the public portal for the purpose of private study or research.

- You may not further distribute the material or use it for any profit-making activity or commercial gain

- You may freely distribute the URL identifying the publication in the public portal 
Rapid Communication

\title{
Low friction thermoplastic polyurethane coatings imparted by surface segregation of amphiphilic block copolymers
}

\author{
Troels Røn ${ }^{\text {a }}$, Irakli Javakhishvili ${ }^{\text {b }}$, Seonghyeon Jeong ${ }^{\text {a }}$, Katja Jankova ${ }^{\text {b, }}$, Seunghwan Lee ${ }^{\text {a,* }}$ \\ ${ }^{a}$ Department of Mechanical Engineering, Technical University of Denmark, DK-2800 Kgs. Lyngby, Denmark \\ ${ }^{\mathrm{b}}$ Danish Polymer Centre, Department of Chemical and Biochemical Engineering, Technical University of Denmark, DK-2800 Kgs. Lyngby, Denmark \\ ${ }^{\mathrm{c}}$ Department of Energy Conversion and Storage, Building 375, Technical University of Denmark, DK-2800 Kgs. Lyngby, Denmark
}

\section{A R T I C L E I N F O}

\section{Keywords:}

Surface lubrication

Amphiphilic block copolymers

Self-segregation

Polymer brushes

Thermoplastic polyurethane (TPU)

\begin{abstract}
A B S T R A C T
This study aims to hydrophilize thermoplastic polyurethane (TPU) surface via selective segregation of amphiphilic block copolymers from bulk TPU matrix, namely "inverted grafting-to" approach. To this end, hydrophilic poly(ethylene glycol) (PEG) polymer chain was employed in either diblock (polycaprolactone- $b$-poly(ethylene glycol) (PCL-b-PEG)) or triblock (poly(ethylene glycol)- $b$-poly(propylene glycol)- $b$-poly(ethylene glycol) (PEG- $b$ PPO-b-PEG)) amphiphilic copolymer additives in polymer matrix. Greatly reduced water contact angles, as low as ca. $13^{\circ}$, from $\geq 90^{\circ}$ for bare substrate proved the successful hydrophilization of TPU surface by this approach, although the degree of hydrophilization was significantly affected by the structure and concentration of embedded amphiphilic copolymers. Moreover, PEG-functionalized TPU surfaces in this approach displayed a reduction of coefficient of friction (COF) by two orders of magnitude. Given that TPU is a widely used material for urinary catheter or other medical devices, we demonstrated a first promising application as lubricious coating to TPU-based urinary catheter tubes.
\end{abstract}

Hydrophobic properties of polymers are advantageous for a number of engineering applications where water or moisture vapor should be kept away. In some contexts, however, hydrophobic properties of polymers rather hinder a better performance such as surface lubricity. Utilization of polymers as sliding bearing materials is typically avoided in aqueous environment due to poor wettability and retention of water, causing failure to form a boundary lubricating film under load, which can result in excessive mechanical wear and energy dissipation [1]. Grafting of hydrophilic polymer brushes has been recognized as an effective means to impart excellent hydrophilicity by attracting and holding water molecules at the solid/water interface [1-6]. Polymer brushes can be formed on surfaces by two main techniques of "graftingto" or "grafting-from" for lubrication purposes [7-9]. Grafting-to method usually comprises adsorption of additive from solution or selective covalent bonding of longer chains to the surface. Grafting-from consists of polymerizing chains out from the surface by feeding monomers to an immobilized initiator on the surface. Some commonly commented drawbacks for these two approaches are either low grafting densities ("grafting-to") $[9,10,11]$ or multi-step preparation ("graftingfrom") via surface initiated polymerization $[4,9,12]$, which may result in poor stability or costly and time-consuming procedures to fabricate the surfaces. Previously, we have presented cross-linked silicone matrices embedded with 10-20 wt\% amphiphilic diblock copolymers, yielding surfaces with coefficients of friction (COF) down to 0.001 in aqueous environment by selective surface segregation - or "inverted grafting-to" - of hydrophilic poly(acrylic acid) (PAA) chains [13]. Selective segregation of hydrophilic chains at the matrix polymer/water interface provides an efficient means to form polymer brushes and to impart surface lubricity [13]. This approach is particularly adequate for applications where a harsh mechanical stress on the surface is acting, e. $\mathrm{g}$. in tribological contacts in bearing systems as it is possible to replenish the polymer brushes from internal matrix even if a firstly formed layer is damaged from tribostress.

In the present study, we challenge to extend the scope of this approach in three aspects. Firstly, in addition to silicone polymer in the previous study [13], we aim to verify the general applicability of this approach by employing another matrix polymer, namely thermoplastic polyurethane (TPU). TPU is utilized in various medical contexts such as urinary catheters, endoscopes, and guide catheters for stents, which often require effective lubrication to reduce discomfort and tissue damage [14,15]. Moreover, while PAA-embedded silicone matrix was added as a coating to substrates such as silicone slab, glass, or stainless

\footnotetext{
* Corresponding author.

E-mail address: seunghwan.lee.jr@gmail.com (S. Lee).
} 
steel [10], TPU has a potential to be used as both the matrix and the substrate too. Secondly, as embedding amphiphilic copolymer with segregating hydrophilic block, we have chosen poly(ethylene glycol) (PEG) that is copolymerized with hydrophobic block polymers, not only in diblock configuration, namely polycaprolactone-block-poly(ethylene glycol) (PCL-b-PEG) (see abstract), but also in a triblock structure, namely a series of 'Poloxamers' (poly(ethylene glycol)- $b$-poly(propylene glycol)- $b$-poly(ethylene glycol) (PEG- $b$-PPO-b-PEG)) (see Fig. 1 and abstract). Lastly, as a first practical application, we utilize this approach to create a lubricious coating onto urinary catheter tubes.

PCL-b-PEG diblock copolymers were purchased from Polymer Source Inc. (Quebec, Canada) and used as received. They are denoted as PCL (X)-b-PEG(Y), where X and Y represent the molecular weights of PCL block and PEG block in $\mathrm{kDa}$, respectively. Examples include PCL(5)-bPEG(5), PCL(5)-b-PEG(10), and PCL(5)-b-PEG(24.5). The polydispersity index (PDI) of the diblock copolymers was $\leq 1.3$. Poloxamers ' $F 127$ ' and 'L121' (Pluronics) were purchased from Sigma-Aldrich and used as received. F127 comprises PEG(4.2)-b-PPO(3.6)-b-PEG(4.2) and L121 PEG(0.2)-b-PPO(3.6)- $b$-PEG(0.2) according to [16]. Stabilized tetrahydrofuran (THF (st.)) $\geq 99 \%$ was purchased from VWR. Thermoplastic polyurethanes (TPU) $11 \mathrm{~T} 80$ and 2363-80AE of Lubrizol were obtained from Bjørn Thorsen A/S (Copenhagen, Denmark). TPUs of $11 \mathrm{~T} 80$ and 2363-80AE are based on PCL and polyether, respectively. Two different types of TPU were utilized to test feasibility of the segregation approach with and without major matrix parts comprising the same hydrophobic chains as copolymer additive, i.e. PCL.

Pellets of $11 \mathrm{~T} 80$ or 2363 -80AE were melted in custom-made Teflon molds in an oven at $180-200{ }^{\circ} \mathrm{C}$ to yield disks ( $30 \mathrm{~mm}$ in diameter and 5 $\mathrm{mm}$ in thickness) after cooling, which were used as substrates. In order to embed amphiphilic copolymers within the TPU matrices, stock solutions of $5 \mathrm{wt} \% 11 \mathrm{~T} 80$ or $2363-80 \mathrm{AE}$ were made first by dissolving and agitating in THF(st.) overnight. These solutions were turbid and viscous, but homogeneous. Block copolymers were then added to the stock TPU solutions to afford $5,10,20$ or $30 \mathrm{wt} \%$ block copolymer dry content, and TPU matrix $11 \mathrm{~T} 80$ or $2363-80 \mathrm{AE}$ accounted for the remaining dry part. For the cases where these solutions were employed as coating, they were added on the TPU substrate by dripping ca. $0.8 \mathrm{~mL}$ of the solution and letting the solvent evaporate overnight. The disks were thus drip-coated with films being ca. $10-15 \mu \mathrm{m}$ in thickness (see Fig. 1 for schematic). The fabricated polymer films were designated as 'Z\%PCL(X)-b-PEG(Y)', where polymer films with $\mathrm{Z}$ wt $\%$ of PCL(X)-b-PEG(Y) in a (100-Z)\% 11 T80 TPU matrix were used. Samples of $10 \%, 20 \%$ or $30 \%$ PEG- $b$-PPO$b$-PEG triblock copolymers in $11 \mathrm{~T} 80$ matrix were made in the same manner and labeled e.g. 'Z\%F127' or 'Z\%L121'. For L121, 20\% sample

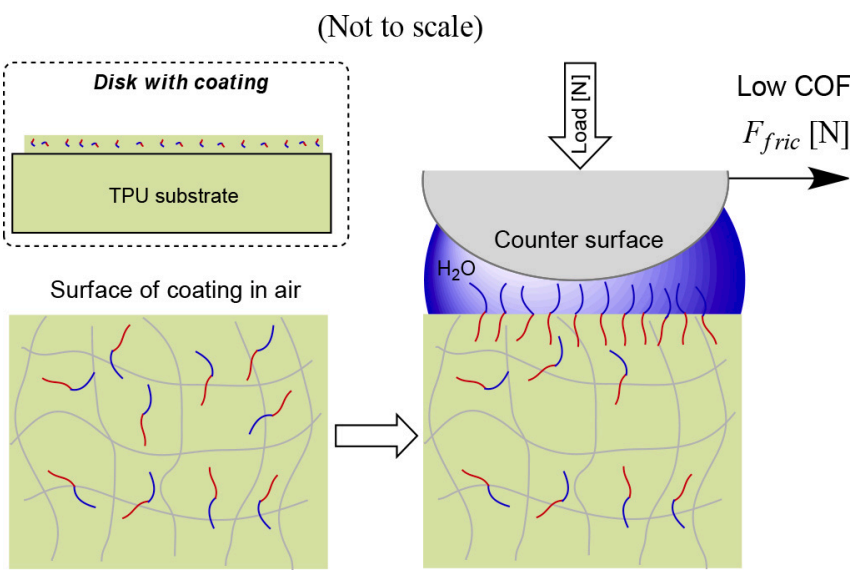

Fig. 1. Schematic representations of i) substrate disk of bulk TPU with coating comprising the same TPU (matrix) and amphiphilic copolymer (additive), ii) close up of coating with matrix TPU blended with diblock copolymers in air (lower left), which surface segregate upon hydration (right). only was prepared. For polymer film samples of 2363-80AE matrix, the name 'Z\%PCL(X)-b-PEG(Y) in 80AE' was chosen. Bulk disks consisting of TPU and embedded amphiphilic copolymer were fabricated by meltmixing $90 \mathrm{wt} \% 11 \mathrm{~T} 80$ and $10 \mathrm{wt} \% \mathrm{~F} 127 \mathrm{in}$ an oven at $200{ }^{\circ} \mathrm{C}$ and casting the bulk disks in Teflon molds and were denoted as '10\%F127-melt'. A photo image of the sample surfaces is shown in the Supplementary Material (Fig. S1). It was attempted to fabricate the bulk disk samples with the wt $\%$ of $80: 20$ and 70:30 for 11T80:F127 (corresponding to '20\%F127-melt' and '30\%F127-melt') too, but they were not mechically robust enough for the purpose of pin-on-disk tribometer experiments (see below).

Wettability by water on the polymer films was characterized with a Ramé-hart goniometer (model 200-F1, Succasunna, NJ) with a software version 2.4.11. A volume of $5 \mu \mathrm{L}$ of MilliQ water was applied to the surfaces and static water contact angles (WCA) were measured after 30 s. Averages and standard deviations of 3-4 measurements were acquired. It should be noted that in 'inverted grafting-to' approach, it is very difficult to determine the grafting density of hydrophilic polymer brush films in a direct manner. Thus, surface wettability by water is not only an evaluation of the functional properties induced by surfacegrafted polymer brushes, but serves as an indirect method to gauge the density/efficacy of grafted polymer brushes as well $[17,18]$. Fig. 2 shows the determined static WCA of the polymer samples. 100\%11 T80 displayed a static WCA of $89.1 \pm 2.7^{\circ}$ that is typical for pristine polymer materials. Samples II-IV in Fig. 2 show the WCAs of the polymer film comprising varied amounts of PCL(5)-b-PEG (5) and $11 \mathrm{~T} 80$ matrix on $11 \mathrm{~T} 80$ substrate disk, namely $5 \%, 10 \%$ and $20 \%$ block copolymer, respectively. The sample 5\%PCL(5)-b-PEG(5) showed also a reduced WCA of $68.2 \pm 2.0^{\circ}$ compared to the reference 11T80, but it was still moderately hydrophobic. Meanwhile, 10\%PCL(5)-b-PEG(5) showed a substantially reduced WCA of $24.3 \pm 2.0^{\circ}$, suggesting a successful selective segregation and exposing of PEG (5) chains to the surface. Lastly, 20\%PCL(5)-b-PEG(5) showed the WCA of $21.9 \pm 1.0^{\circ}$ that was almost indistinguishable from 10\%PCL(5)-b-PEG(5). These observations suggest that the selective segregation of PEG (5) chains from the $11 \mathrm{~T} 80$ matrix is saturated at $10 \%$ block copolymer content of PCL(5)-b-PEG (5).

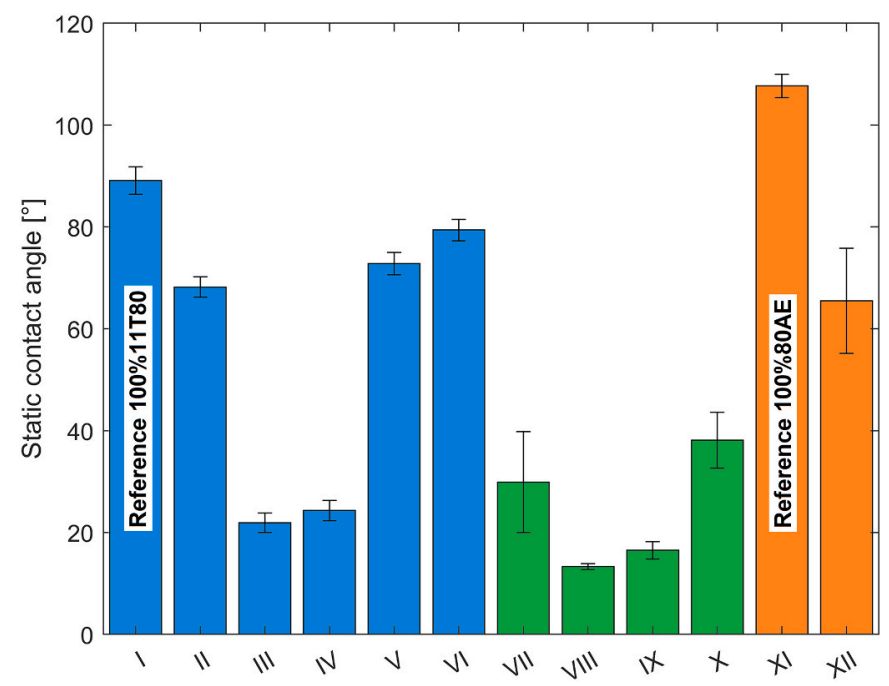

Fig. 2. Static water contact angle (WCA) of produced surfaces. Values are averages of 3 measurements, error bars indicate standard deviation. Turquoies bars: PCL-based matrix and PCL-based diblocks additives, Green bars: PCLbased matrix and poloxamer-based triblock additives, Orange: polyetherbased matrix and PCL-based diblocks. I: 100\%11T80, II: 5\%PCL(5)-b-PEG (5), III: 10\%PCL(5)-b-PEG(5), IV: 20\%PCL(5)-b-PEG(5), V: 20\%PCL(10)-bPEG(5), VI: 20\%PCL(24.5)-b-PEG(5), VII: 10\%F127-melt, VIII: 20\%F127, IX: 30\%F127, X: 20\%L121, XI: 100\%80AE, XII: 20\%PCL(5)-b-PEG(5) in 80AE. (For interpretation of the references to colour in this figure legend, the reader is referred to the web version of this article.) 
Comparison of samples IV, V and VI in Fig. 2 shows the influence of PCL block size on the water wettability of the yielded films. The WCAs were $24.3 \pm 2.0^{\circ}, 72.8 \pm 2.2^{\circ}$ and $79.4 \pm 2.1^{\circ}$ for $20 \%$ PCL $(X)$ - $b$-PEG (5), where $X$ is 5 (IV), $10(\mathrm{~V})$, and 24.5 (VI), respectively. These values indicate that the mobility of $20 \% \operatorname{PCL}(X)$-b-PEG (5) within the matrix is severely hampered when the molecular weight of PCL block is $10 \mathrm{kDa}$ or higher.

Static WCAs for polymer film samples of 10\%F127-melt, 20\%F127, 30\%F127 and 20\%L121 are displayed in Fig. 2 too. 20\%F127 (VIII) and 30\%F127 (IX in Fig. 2) were very hydrophilic with WCAs of $13.3 \pm 0.6^{\circ}$ and $16.5 \pm 1.7^{\circ}$, which indicates an effective migration and segregation of the PEG chains on the matrix surface for these triblock copolymers as well. 10\%F127-melt (VII in Fig. 2) showed somewhat higher WCA at $29.9 \pm 9.9^{\circ}$. We ascribe it to the lower F127 concentration of $10 \%$ F127melt and inhomogeneity of the film when water comes in contact with the surface. Similar trends have been reported by Lee et al. who utilized segregation of poloxamers from TPU matrix at the polymer/water interface, but did not investigate the surfaces' tribological properties $[14,19]$. The WCA of 20\%L121 (X in Fig. 2) was $38.1 \pm 5.5^{\circ}$. When comparing F127 and L121, the molecular weight of hydrophobic blocks is $3.6 \mathrm{kDa}$ for both, but that of PEO blocks are vastly different, namely $0.2 \mathrm{kDa}(\mathrm{L} 121)$ and $4.2 \mathrm{kDa}$ (F127), and it is likely to be responsible for a superior hydrophilicity of $20 \% \mathrm{~F} 127$.

Lastly, the influence of the matrix was investigated by employing 2 different TPUs, $11 \mathrm{~T} 80$ and $80 \mathrm{AE}$ as the matrix substrate, having different structure and 20\%PCL(5)-b-PEG(5) as the embedded amphiphilic copolymer. 11T80 is a TPU based on PCL segments, whereas 80AE is based on polyether-type polytetrahydrofuran, poly(THF). Thus, the point of interest was whether PCL(5)-b-PEG (5) can migrate in a matrix of $80 \mathrm{AE}$, where the hydrophobic soft segments are not the same type. 20\%PCL(5)-b-PEG(5) in 80AE showed a clear reduction in the WCA $\left(65.5 \pm 10.3^{\circ}, \mathrm{XII}\right.$ in Fig. 2) compared to $100 \% 80 \mathrm{AE}$ substrate $(107 \pm$ $2.3^{\circ}, \mathrm{XI}$ in Fig. 2). This undoubtedly indicates that PCL(5)-b-PEG (5) can migrate in the 80AE matrix to some extent, albeit the resulting surface is not as hydrophilic as the 20\%PCL(5)-b-PEG(5) in $11 \mathrm{~T} 80$ matrix (24.3 \pm $\left.2.0^{\circ}\right)$.

The lubricating capabilities of the drip-coated films and bulk disks in an aqueous environment were characterized with a pin-on-disk tribometer (CSM Instruments with 4.4 M software version). Details on the operational principles of the tribometer are available in previous publications [13]. Unless otherwise stated, the slider (pin) was smooth PDMS (hemispherical, with $3 \mathrm{~mm}$ radius at the apex). The calculated contact pressures between polyurethane disk (ca. 4.0 MPa Young's modulus) and a PDMS pin (2.0 MPa Young's modulus) or 316 steel ball (190 GPa Young's modulus) were $0.34 \mathrm{MPa}$ or $0.71 \mathrm{MPa}$, respectively (pin or ball with the diameter $6 \mathrm{~mm}$ ). The experiments were conducted at room temperature, recording the mean $\operatorname{COF}\left(=F_{\text {friction }} /\right.$ Load $)$ values over a minimum of 20 laps for each speed measured with standard normal load of $2 \mathrm{~N}$. Error bars in $\mathrm{COF}$ vs sliding speed plots designate the standard deviation. Longer-term lubricity tests with pin-on-disk were also performed with PDMS pins and TPU substrate with $10 \mathrm{~N}$ load $(0.57$ MPa contact pressure) over 100,000 laps.

Fig. 3(a) displays the tribological testing results of $11 \mathrm{~T} 80 \mathrm{TPU}$ surfaces with varying amount of PCL(5)-b-PEG (5) in PBS at pH 7.4. 20\% PCL(5)-b-PEG(5) and 10\%PCL(5)-b-PEG(5) showed good lubricating properties with $\mathrm{COF} \approx 0.03$ at higher sliding speeds $(25-100 \mathrm{~mm} / \mathrm{s})$ and gradually lowering COF down to $0.02-0.01$ at the lowest sliding speed. Reference $11 \mathrm{~T} 80$ TPU surface resulted in COF $\geq 1$ throughout the entire range of tested sliding speeds. Thus, incorporation of 10 and 20\%PCL (5)-b-PEG (5) in the TPU film reduces the friction of the TPU surface by a factor of 50-100. 5\%PCL(5)-b-PEG(5) also showed a good lubricity in the high speed range, $>25 \mathrm{~mm} / \mathrm{s}$, however, at lower sliding speeds, the COFs were ca. 0.1 , which suggests poor boundary lubricating capabilities. The pin-on-disk tribometry results of 5, 10, and 20\%PCL(5)-b-PEG (5) samples (Fig. 3(a)) corroborate well the WCA data (Fig. 2) that showed the inferior surface hydrophilicity by $5 \%$ PCL(5)-b-PEG(5) compared to $10 \%$ PCL(5)-b-PEG(5) and 20\%PCL(5)-b-PEG(5). Additionally, 20\%PCL(5)-b-PEG(5) displayed low COFs, 0.02-0.06, when slid against a stainless steel pin despite a higher apparent contact pressure $(0.71 \mathrm{MPa})$ than PDMS pin $(0.34 \mathrm{MPa})$ and higher surface roughness (Fig. 4). The influence of PCL block length on the lubricity of the films was also investigated, and the results are shown in Fig. 3(b). 20\% PCL(5)-b-PEG(5) showed a good lubricity (data reproduced from Fig. 3 (a)), whereas $20 \%$ PCL(10)-b-PEG(5) showed a mediocre lubricity at the very highest sliding speeds. Finally, 20\%PCL(24.5)-b-PEG(5) did not show any friction reduction (the COFs data were collected at 1,10 and $100 \mathrm{~mm} / \mathrm{s}$ only) compared to reference $11 \mathrm{~T} 80 \mathrm{TPU}$. Thus, the COF vs speed plots in Fig. 3(b) also corroborate very well the wettability by observed WCAs shown in Fig. 2. These results indicate that increase of PCL block size adversely affects surface segregation and probably limits migration in matrix polymer.

Poloxamers F68 and F127 have been previously reported as aqueous lubricant additives by showing adsorption at the interface of the sliding contact [20-22]. The hydrophilic PEG chains and the poloxamers' ability
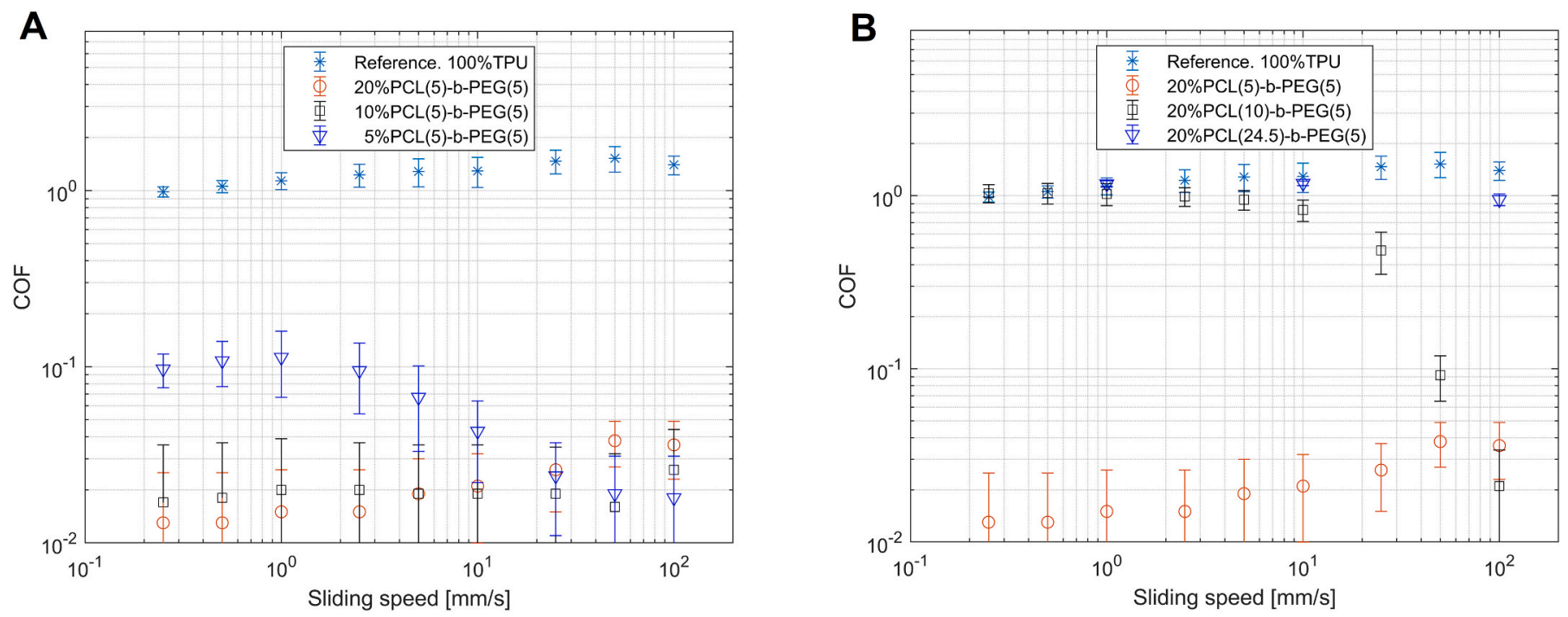

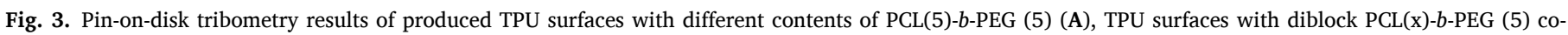
polymers of different PCL block size (B). Error bars indicate standard deviation. 


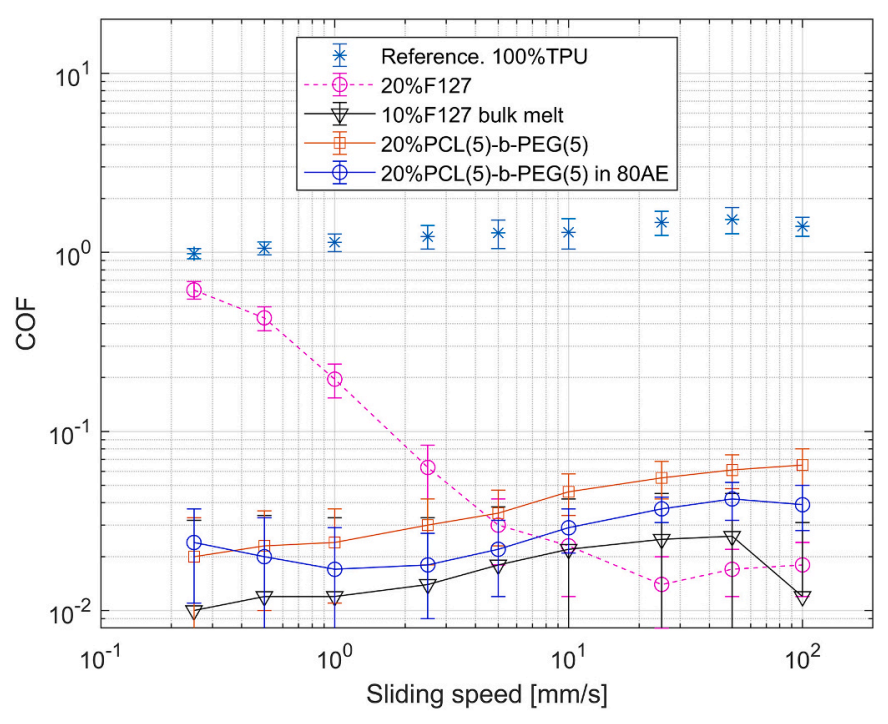

Fig. 4. Pin-on-disk tribometry results of F127 and PCL(5)-b-PEG (5) containing TPU surfaces, in 80AE matrix and 316 steel pin (red squares). Lines connecting the points are a guide to the eye. Error bars indicate standard deviation. (For interpretation of the references to colour in this figure legend, the reader is referred to the web version of this article.)

to gel and adsorb on various surfaces are some of the properties to be responsible for them functioning as aqueous lubricant additives [17]. In this study, we have investigated the lubricity of F127 embedded in 11 T80 matrix as films or in bulk melt. As shown in Fig. 4, the COFs of 20\%F127 were at the level of 0.02-0.04 in high-to-medium sliding speeds $(100-5 \mathrm{~mm} / \mathrm{s})$, but gradually increased to ca. 0.6 with lowered sliding speed. The tribological behavior of $10 \% \mathbf{F 1 2 7}$ was similar to that of 20\%F127 in that the COF was very low only at the highest speed (100 $\mathrm{mm} / \mathrm{s}$ ), but rapidly increased in medium-to-low speed regime (data not shown). Unlike the cases of PCL-b-PEG above, the correlation between surface wettability and lubricity is not excellent. The very low WCA, $13.3 \pm 0.6^{\circ}$, yet a limited lubricity of $20 \% \mathrm{~F} 127$ suggests that while PEG chains were initially formed at a high grafting density, some of them might be removed from tribological stress during pin-on-disk tribometry experiments. In a control experiment, the test buffer solution of $\mathbf{2 0 \%}$ F127 film was collected and another tribological experiment was conducted between a new pristine $\mathbf{1 0 0 \% 1 1 T 8 0}$ disk and a PDMS pin using this buffer solution. This resulted in intermediate COF values between the reference disk and the 20\%F127 film (data not shown). We interpret this behavior as leaching of F127 into the solution under tribostress from the initial experiment, where leached F127 contributes to the lubricity. In contrast, no leaching of PCL(5)-b-PEG(5) into solution was indicated from the same type of control experiments. The molecular weight/hydrophobicity of the PCL(5) block are apparently sufficient for it to act as a stable and effective anchoring block at the water/matrix interface without leaching, whereas those of $\mathrm{PPO}(3.6)$ in $\mathrm{F} 127$ are not.

Fig. 4 also includes pin-on-disk testing results of a disk 10\%F127melt, displaying a good lubricity throughout the entire sliding speed range. This is in a stark contrast to $20 \% \mathbf{F 1 2 7}$, where an effective lubrication was observed in high-speed regime only. Although wt $\%$ of F127 is higher in 20\%F127 than 10\%F127-melt, and the WCA for $\mathbf{2 0 \%}$ F127 is distinctly lower than that of 10\%F127-melt (Fig. 2), the embedded F127 is present in a ca. $10-15 \mu \mathrm{m}$ thick coating only in the former, whereas it is present within the entire disk block (ca. $5 \mathrm{~mm}$ in thickness) in the latter. Thus, ample amount of available F127 within TPU matrix and continuous replenishment at the surface following tribostress-induced loss appear to be driving the superior lubricity of 10\%F127-melt. This observation also suggests that the correlation between water wettability and lubricity is not necessarily proportional to each other when the segregated polymers chains are not stable under tribostress; while the wettability by water is mainly determined by the initial surface hydrophilicity of polymer-covered surface, it is more important to maintain the surface hydrophilicity for effective lubricity in water. In fact, a minor degree of turbidity of the solution was observed after the tribological tests of 10\%F127-melt. Sample 20\%L121 had very poor boundary lubricity with COFs in the range $0.5-0.9$ in medium and low sliding speeds range, only with some lubricity $(\mathrm{COF}=0.01)$ at the very highest sliding speed of $100 \mathrm{~mm} / \mathrm{s}$ (data not shown). The short length PEG chains $(0.2 \mathrm{kDa})$ seem insufficient to form lubricious water film, which is consistent with its mediocre wettability compared to F127 (Fig. 2).

Lastly, we have investigated the longer term lubricity of $20 \%$ PCL(5)b-PEG(5) where the surface was slid against PDMS pin at $10 \mathrm{~N}$ load. The results are displayed in Supplementary Material (Fig. S4). The 20\%PCL (5)-b-PEG(5) film maintained its lubricity with COF $=0.01-0.02$ for 100,000 labs $(>10 \mathrm{~h}$ ) despite extensive tribostress.

As a first practical application of this approach, uncoated pristine PU urinary catheters were dip-coated in a solution of ' $30 \% \mathrm{~F} 127$ ' with a total dry polymer content of $5-6 \mathrm{wt} \%$ (Fig. S2). The catheters were dried overnight after dip coating to render a ca. $30 \mu \mathrm{m}$ thick film (see SEM image in Fig. S3). The slipperiness of urinary catheters was evaluated with in-vitro PDMS-based urethra model and reciprocal motion friction tester; the details are found in a recent paper [23] and its schematic representation in Fig. S5. Briefly, PDMS-based model urethras were with a duct through the center of cylindrical model urethra, where catheters can be inserted for simulated catheterization. Soft PDMS ducts were materialized by casting and curing Sylgard 527/184 (Dow Corning) mixed in 20/1 wt. ratio. The duct diameter was $3.75 \mathrm{~mm}$ whereas tested catheters were $4.7 \mathrm{~mm}$ in diameter. The catheters' diameter, larger than that of the soft PDMS duct, was selected in order to emulate the resistance from opening the urinary tract's mucosal folds. A thin steel rod was inserted first as guide wire into the catheters to minimize buckling of catheter tubes during insertion. Catheters were pre-wetted in PBS pH 7.4 for 2-3 s prior to tests. Friction tests were performed under immersion of PDMS model urethras in PBS pH 7.4.

Fig. 5 displays the measured forces (average of 4 cycles) from the invitro catheter friction tester. To provide a reference for an uncoated catheter, a PVC catheter with a diameter of $4.7 \mathrm{~mm}$ was attempted to be inserted into the PDMS duct, but the test failed due to high friction resistance (force $>1 \mathrm{~N}$ ) and resulting buckling of catheter tubes. Fig. 5 shows the observed force cycles of the 30\%F127 coating versus commercially available 'Curicat' (Curion, Mediq, Denmark) catheter with hydrophilic polyvinylpyrrolidone (PVP) coating - during simulated catheterization. It is emphasized that the forces observed in the catheter tests are not solely caused by friction, but can be a combination of elastic opening of the duct during insertion, surface friction, and strain forces [23]. PU catheters coated with $\mathbf{3 0 \%} \% \mathbf{1 2 7}$-coating yielded peak forces $(0.25 \mathrm{~N})$, still larger than Curicat catheters during insertion $(0.08 \mathrm{~N})$, but represent substantially lower forces than the uncoated reference. The average peak forces during retraction were $0.05 \mathrm{~N}$ for Curicat and $0.10 \mathrm{~N}$ for 30\% 127 -coating, where plateau forces were $0.01 \mathrm{~N}$ and $0.07 \mathrm{~N}$, respectively. The $30 \% \mathbf{F 1 2 7}$ coating is reasonably lubricious compared to the PVP-coated Curicat catheters albeit observed peak forces during insertion gave the largest differences. The friction tester results of catheters 30\%F127 display the film's lubricity and a potential application for urinary catheters or other tubular medical entities.

In summary, this study has shown successful surface functionalization of TPU via selective segregation of amphiphilic block copolymers from bulk TPU matrix to impart hydrophilicity to the polymer/water interface and reduce COF up to two orders of magnitude. The observed lubricity was generally well corroborated with wettability. One of the main factors to determine the segregation of the amphiphilic PCL- $b$-PEG diblock copolymers to the matrix polymer/water interface was observed to be the molecular weight of PCL block. PCL- $b$-PEG diblock copolymers yielded stable lubricity without leaching, whereas TPU films with poloxamer F127 were observed to passably leach. TPU films containing 

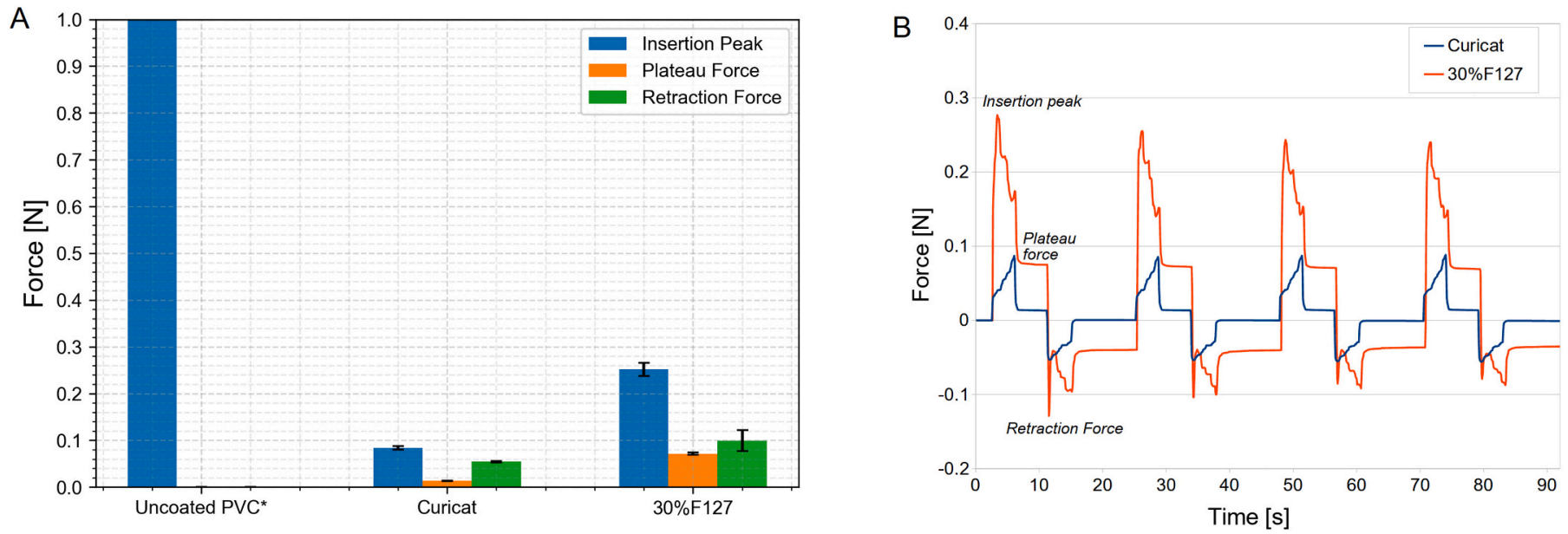

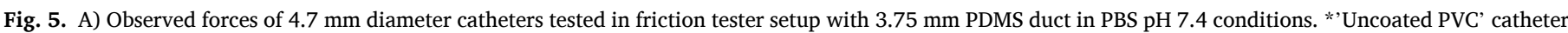

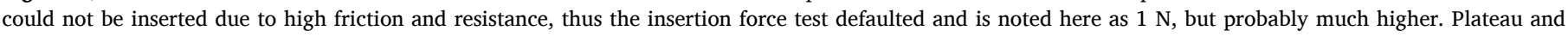

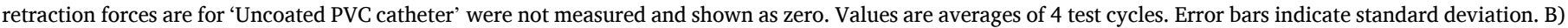
Observed forces with reciprocal motion friction tester in PDMS model urethras. Four cycles with observed 'insertion', 'plateau' and 'retraction' forces.

F127 also showed potential to lubricate tubular medical devices such as urinary catheters in aqueous environment.

\section{Author credit statement}

Troels Røn: Conceptualization, Methodology, Data Curation, Formal analysis, Draft Writing, Editing, Writing original draft.

Irakli Javakhishvili: Conceptualization, Writing - review \& editing. Seonghyeon Jeong: Conceptualization, Methodology, Data Curation, Writing - review \& editing.

Katja Jankova: Conceptualization, Writing - review \& editing.

Seunghwan Lee: Conceptualization, Project administration, Methodology, Writing - review \& editing.

\section{Declaration of Competing Interest}

The authors have NO conflict of interest to declare.

\section{Acknowledgments}

We greatly appreciate the donation of TPU polymers ' 11 T80' and '2363-80AE' from Lubrizol and their Danish distributor contact Svend Rimestad of Bjørn Thorsen A/S (Denmark). Appreciation is bestowed upon the Independent Research Fund Denmark (DFF), Individual Postdoc Grant, Project No. 5054-00161B and ERC Starting Grant 2010 (project no. 261152) for funding. We declare no financial conflict of interest.

\section{Appendix A. Supplementary data}

Supplementary data to this article can be found online at https://doi. org/10.1016/j.colcom.2021.100477.

\section{References}

[1] J. Klein, Hydration lubrication, Friction 1 (1) (2013) 1-23.

[2] U. Raviv, S. Giasson, N. Kampf, J.F. Gohy, R. Jérôme, J. Klein, Lubrication by charged polymers, Nature 425 (6954) (2003) 163-165.

[3] F. Goujon, A. Ghoufi, P. Malfreyt, D.J. Tildesley, The kinetic friction coefficient of neutral and charged polymer brushes, Soft Matter 9 (10) (2013) 2966-2972.

[4] A. Nomura, K. Ohno, T. Fukuda, T. Sato, Y. Tsujii, Lubrication mechanism of concentrated polymer brushes in solvents: effect of solvent viscosity, Polym. Chem. 3 (1) (2012) 148-153.
[5] J.K. Riley, K. Matyjaszewski, R.D. Tilton, Friction and adhesion control between adsorbed layers of polyelectrolyte brush-grafted nanoparticles via pH-triggered bridging interactions, J. Colloid Interface Sci. 526 (2018) 114-123.

[6] Q. Wei, X. Pei, J. Hao, M. Cai, F. Zhou, W. Liu, Surface modification of diamondlike carbon film with polymer brushes using a bio-inspired catechol anchor for excellent biological lubrication, Adv. Mater. Interfaces 1 (5) (2014) 1400035.

[7] K. Chawla, S. Lee, B.P. Lee, J.L. Dalsin, P.B. Messersmith, N.D. Spencer, A novel low-friction surface for biomedical applications: modification of poly (dimethylsiloxane)(PDMS) with polyethylene glycol (PEG)-DOPA-lysine, J. Biomed. Mater. Res. A 90 (3) (2009) 742-749.

[8] B. Liberelle, S. Giasson, Friction and normal interaction forces between irreversibly attached weakly charged polymer brushes, Langmuir 24 (4) (2008) 1550-1559.

[9] R. Heeb, R.M. Bielecki, S. Lee, N.D. Spencer, Room-temperature, aqueous-phase fabrication of poly (methacrylic acid) brushes by UV-LED-induced, controlled radical polymerization with high selectivity for surface-bound species, Macromolecules 42 (22) (2009) 9124-9132.

[10] B. Zdyrko, I. Luzinov, Polymer brushes by the "grafting to" method, Macromol. Rapid Commun. 32 (12) (2011) 859-869.

[11] C. Ligoure, L. Leibler, Thermodynamics and kinetics of grafting end-functionalized polymers to an interface, J. Phys. 51 (12) (1990) 1313-1328.

[12] J. Yang, H. Chen, S. Xiao, M. Shen, F. Chen, P. Fan, M. Zhong, J. Zheng, Saltresponsive zwitterionic polymer brushes with tunable friction and antifouling properties, Langmuir 31 (33) (2015) 9125-9133.

[13] T. Røn, I. Javakhishvili, S. Hvilsted, K. Jankova, S. Lee, Ultralow friction with hydrophilic polymer brushes in water as segregated from silicone matrix, Adv. Mater. Interfaces 3 (2) (2016) 1500472.

[14] J.H. Lee, Y.M. Ju, W.K. Lee, K.D. Park, Y.H. Kim, J. Biomed. Mater. Res. 40 (2) (1998) 314-323.

[15] R.J. Zdrahala, I.J. Zdrahala, Biomedical applications of polyurethanes: a review of past promises, present realities, and a vibrant future, J. Biomater. Appl. 14 (1) (1999) 67-90.

[16] P. Alexandridis, T.A. Hatton, Colloids Surf. A Physicochem. Eng. Asp. 96 (1-2) (1995) 1-46.

[17] Manabu Inutsuka, et al., High density polymer brush spontaneously formed by the segregation of amphiphilic diblock copolymers to the polymer/water interface, ACS Macro Lett. 2 (3) (2013) 265-268.

[18] M. Inutsuka, H. Tanoue, N.L. Yamada, K. Ito, H. Yokoyama, Dynamic contact angle on a reconstructive polymer surface by segregation, RSC Adv. 7 (28) (2017) $17202-17207$.

[19] J.H. Lee, Y.M. Ju, D.M. Kim, Platelet adhesion onto segmented polyurethane film surfaces modified by addition and crosslinking of PEO-containing block copolymers, Biomaterials 21 (7) (2000) 683-691.

[20] S. Lee, R. Iten, M. Müller, N.D. Spencer, Influence of molecular architecture on the adsorption of poly (ethylene oxide)- poly (propylene oxide)- poly (ethylene oxide) on PDMS surfaces and implications for aqueous lubrication, Macromolecules 37 (22) (2004) 8349-8356.

[21] S. Lee, N.D. Spencer, Aqueous lubrication of polymers: influence of surface modification, Tribol. Int. 38 (11-12) (2005) 922-930.

[22] T. Røn, I.S. Chronakis, S. Lee, Lubrication of soft and hard interfaces with thermoresponsive F127 hydrogel, Polymer 55 (22) (2014) 5708-5717.

[23] T. Røn, K.P. Jacobsen, S. Lee, A catheter friction tester using balance sensor: combined evaluation of the effects of mechanical properties of tubing materials and surface coatings, J. Mech. Behav. Biomed. Mater. 84 (2018) 12-21. 